\title{
Thermal rectification effects of multiple semiconductor quantum dot junctions
}

\author{
David M.-T. Kuo \\ Department of Electrical Engineering and Department of Physics, \\ National Central University, Chungli, 320 Taiwan
}

(Dated: December 6, 2018)

\begin{abstract}
Based on the multiple energy level Anderson model, this study theoretically examines the thermoelectric effects of semiconductor quantum dots (QDs) in the nonlinear response regime. The charge and heat currents in the sequential tunneling process are calculated by using the Keldysh Green's function technique. Results show that the thermal rectification effect can be observed in a multiple QD junction system, whereas the tunneling rate, size fluctuation, and location distribution of QD significantly influence the rectification efficiency.
\end{abstract}

\section{INTRODUCTION}

Due to global warming and environment issues, it is important to developing the new energies to reduce the total amount of $\mathrm{CO}_{2}$ in the earth's atmosphere. Electrical power generated by the mechanical force of wind and ocean tides is one of several types of green energy. Apart from that, electrical power generated from solar energy transformation also creates clean energy. Researchers have used many technologies to develop materials with high values of figure of merit (ZT) for potential applications of solid-state thermal devices. These materials can be utilized to generate electrical power through the temperature bias resulting from solar heating or other method heating.[1-9]

Researcher have proposed several methods for enhancing ZT.[2] One of them is to reduce system dimensions.[3] A zero dimension QD system was predicted to be more pronounced for the enhancement of thermoelectric efficiency in the dimension reduction.[9] Nevertheless, previous studies focus on the linear response regime of $\Delta T / T_{0} \ll 1$, where $\Delta T$ and $T_{0}$ are the temperature bias and equilibrium temperature, respectively, of two side electrodes.[10] The properties of thermal devices in the nonlinear response regime $\Delta T / T_{0} \sim 1$ have important applications such as thermal rectifiers and thermal transistors. A thermal rectifier is crucial for the heat current storage. Records of thermal rectification date back as early as 1935, when Starr discovered that copper oxide/copper junctions can exhibit a thermal diode behavior.[11] The thermal rectification effects have been theoretically predicted to occur in one dimensional phonon junction systems.[12-15] Because phonons are heat flow carriers in refs [12-15], their applications are restricted to the heat manipulation and heat energy storage. Using charge carriers (electrons or holes), we can manipulate heat currents and charge currents. The Peltier effect with the application of coolers makes it possible to manipulate the heat current using applied voltage. Its reverse process is so called the Seebeck effect used to create electrical power.

Scheibner and coworkers have, recently, employed a single metallic QD junction to measure electrochemical potential in the linear response regime.[16] Such a small electrochemical potential yielded by temperature bias is not sufficient to demonstrate the thermal rectification effect.[17] Besides, the operation temperature of a metallic QD is very low because its charging energies are much smaller than the thermal energy $\left(k_{B} T_{0}=\right.$ $25 m e V)$. Semiconductor QD junctions with large interdot Coulomb interactions and energy-level separation within each QD allow semiconductor QD thermal rectifiers to operate at much higher temperatures. Previous studies of refs[16,17] only consider a single QD junction, which is useful for the case of low QD density. To scale up QD thermal devices, it is necessary to consider the case of high QD density.

This study demonstrates that a system of multiple semiconductor QDs embedded in an amorphous insulator with low heat conductivity connected to the metallic electrodes exhibits the thermal rectification effect in the nonlinear response regime. This study also clarify the thermal power in the linear and nonlinear regimes. Although the mechanism of thermal rectification for QD junctions is similar to that of charge current, the heat current is yielded by temperature bias and electrochemical potential. The very nonlinear relation between the applied temperature bias and the electrochemical potential leads to that it is not straightforward to reveal the relation between the heat current and the applied temperature bias.

\section{FORMALISM}

The following multi-level Anderson model [18] describes the Hamiltonian of the metal/quantum dots/metal double barrier junction in Fig. 1 ;

$$
\begin{aligned}
H & =\sum_{k, \sigma, \beta} \epsilon_{k} a_{k, \sigma, \beta}^{\dagger} a_{k, \sigma, \beta}+\sum_{\ell, \sigma} E_{\ell} d_{\ell, \sigma}^{\dagger} d_{\ell, \sigma} \\
& +\sum_{\ell, \sigma} U_{\ell} d_{\ell, \sigma}^{\dagger} d_{\ell, \sigma} d_{\ell,-\sigma}^{\dagger} d_{\ell,-\sigma}+\frac{1}{2} \sum_{\ell \neq j ; \sigma, \sigma^{\prime}} U_{\ell, j} d_{\ell, \sigma}^{\dagger} d_{\ell, \sigma} d_{j, \sigma^{\prime}}^{\dagger} d_{j, \sigma^{\prime}} \\
& +\sum_{k, \sigma, \beta, \ell} V_{k, \beta, \ell} a_{k, \sigma, \beta}^{\dagger} d_{\ell, \sigma}+\sum_{k, \sigma, \beta, \ell} V_{k, \beta, \ell}^{*} d_{\ell, \sigma}^{\dagger} a_{k, \sigma, \beta}
\end{aligned}
$$


where $a_{k, \sigma, \beta}^{\dagger}\left(a_{k, \sigma, \beta}\right)$ creates (destroys) an electron of momentum $k$ and spin $\sigma$ with energy $\epsilon_{k}$ in the $\beta$ metallic electrode. The term $d_{\ell, \sigma}^{\dagger}\left(d_{\ell, \sigma}\right)$ creates (destroys) an electron with the ground-state energy $E_{\ell}$ of $\ell$ th QD , while $U_{\ell}$ and $U_{\ell, j}$ describe, respectively, the intradot Coulomb interactions and the interdot Coulomb interactions. The term $V_{k, \beta, \ell}$ describes the coupling between the band states of electrodes and the QDs. The Hamiltonian of Eq. (1) assumes that energy level separations between the ground state and the first excited state within each QD is much larger than intradot Coulomb interactions $U_{\ell}$ and thermal temperature $k_{B} T$. Therefore, there is only one energy level for each QD. This study ignores the interdot hopping terms due to the high potential barrier separating QDs.

Using the Keldysh-Green's function technique,[19] the charge and heat currents leaving electrodes can be expressed as

$$
\begin{gathered}
J_{e}=\frac{-2 e}{h} \sum_{\ell} \int d \epsilon \gamma_{\ell}(\epsilon) \operatorname{Im} G_{\ell, \sigma}^{r}(\epsilon) f_{L R}(\epsilon), \\
Q=\frac{-2}{h} \sum_{\ell} \int d \epsilon \gamma_{\ell}(\epsilon) \operatorname{Im} G_{\ell, \sigma}^{r}(\epsilon)\left(\epsilon-E_{F}-e \Delta V\right) f_{L R}(\epsilon),
\end{gathered}
$$

where $f_{L R}(\epsilon)=f_{L}(\epsilon)-f_{R}(\epsilon)$, the transmission factor is $\gamma_{\ell}(\epsilon)=\frac{\Gamma_{\ell, L}(\epsilon) \Gamma_{\ell, R}(\epsilon)}{\Gamma_{\ell, L}(\epsilon)+\Gamma_{\ell, R}(\epsilon)}, f_{L(R)}(\epsilon)=$ $1 /\left(\exp ^{\left(\epsilon-\mu_{L(R)}\right) /\left(k_{B} T_{L(R)}\right)}+1\right)$ is the Fermi distribution functions for the left (right) electrode. The chemical potential difference between these two electrodes is related to the bias difference $\mu_{L}-\mu_{R}=e \Delta V$. The temperature difference is $T_{L}-T_{R}=\Delta T$, and $E_{F}$ is the Fermi energy of electrodes. $\Gamma_{\ell, L}(\epsilon)$ and $\Gamma_{\ell, R}(\epsilon)$ $\left[\Gamma_{\ell, \beta}=2 \pi \sum_{\mathbf{k}}\left|V_{\ell, \beta, \mathbf{k}}\right|^{2} \delta\left(\epsilon-\epsilon_{\mathbf{k}}\right)\right]$ denote the tunneling rates from the QDs to the left and right electrodes, respectively. Notations $e$ and $h$ denote the electron charge and Plank's constant, respectively. For simplicity, we assumed that these tunneling rates are energy- and biasindependent. Equations (2) and (3) have been employed to reveal the thermal properties of a single-level QD in the Kondo regime.[20,21] Here, this analysis focuses on the heat current in the Coulomb blockade regime. Previous studies $[22,23]$ provide an expression of the retarded Green function

$$
\begin{aligned}
G_{\ell, \sigma}^{r}(\epsilon) & =\left(1-N_{\ell-\sigma}\right) \sum_{m=1}^{3^{n-1}} \frac{p_{m}}{\epsilon-E_{\ell}-\Pi_{m}+i \Gamma_{\ell}} \\
& +N_{\ell-\sigma} \sum_{m=1}^{3^{n-1}} \frac{p_{m}}{\epsilon-E_{\ell}-U_{\ell}-\Pi_{m}+i \Gamma_{\ell}},
\end{aligned}
$$

where $n$ denotes the number of QDs. The term $\Pi_{m}$ denotes the sum of Coulomb interactions seen by a particle in $\operatorname{dot} \ell$ due to other particles in the $\operatorname{dot} j(j \neq \ell)$, which can be occupied by zero, one or two particles. The probability factor $p_{m}$ denotes the probability of such configurations. For a three-QD system $\left(\ell \neq j \neq j^{\prime}\right)$, there are nine
$(3 \times 3)$ configurations, and the probability factors become $p_{1}=a_{j} a_{j^{\prime}}, p_{2}=b_{j} a_{j^{\prime}}, p_{3}=a_{j} b_{j^{\prime}}, p_{4}=c_{j} a_{j^{\prime}}, p_{5}=c_{j^{\prime}} a_{j}$, $p_{6}=b_{j} b_{j^{\prime}}, p_{7}=c_{j} b_{j^{\prime}}, p_{8}=c_{j^{\prime}} b_{j}$, and $p_{9}=c_{j} c_{j^{\prime}}$, where $a_{j}=1-\left(N_{j, \sigma}+N_{j,-\sigma}\right)+c_{j}, b_{j}=\left(N_{j, \sigma}+N_{j,-\sigma}\right)-2 c_{j}$, and $c_{j}=\left\langle n_{j,-\sigma} n_{j, \sigma}\right\rangle$ is the intradot two particle correlation function. $N_{j, \sigma}$ is one particle occupation number. The interdot Coulomb interaction factors are $\Pi_{1}=0$, $\Pi_{2}=U_{\ell, j}, \Pi_{3}=U_{\ell, j^{\prime}}, \Pi_{4}=2 U_{\ell, j}, \Pi_{5}=2 U_{\ell, j^{\prime}}$, $\Pi_{6}=U_{\ell, j}+U_{\ell, j^{\prime}}, \Pi_{7}=2 U_{\ell, j}+U_{\ell, j^{\prime}}, \Pi_{8}=2 U_{\ell, j^{\prime}}+U_{\ell, j}$, and $\Pi_{9}=2 U_{\ell, j}+2 U_{\ell, j^{\prime}}$. The sum of probability factors $p_{m}$ for all configurations equals to 1 , indicating the fact that $G_{\ell, \sigma}^{r}(\epsilon)$ satisfies the sum rule. The imaginary part of each resonant channel is $\left.\Gamma_{\ell}=\left(\Gamma_{\ell, L}+\Gamma_{\ell, R}\right)\right) / 2$ resulting from the coupling between the QDs and the electrodes, where the real part of self energy is ignored. A self energy that ignores the effects of electron Coulomb interactions is adequate in the Coulomb blockade regime, but it does not capture the Kondo effect.

According to the expression of the retarded Green's function in Eq. (4), we need to know the occupation numbers $N_{\ell, \sigma}\left(N_{\ell,-\sigma}\right)$ and $N_{\ell, \ell}=c_{\ell}$. These numebrs can be obtained by solving the following equations selfconsistently:

$$
\begin{gathered}
N_{\ell, \sigma}=-\int \frac{d \epsilon}{\pi} \frac{\Gamma_{\ell, L} f_{L}(\epsilon)+\Gamma_{\ell, R} f_{R}(\epsilon)}{\Gamma_{\ell, L}+\Gamma_{\ell, R}} \operatorname{Im} G_{\ell, \sigma}^{r}(\epsilon) \\
c_{\ell}=-\int \frac{d \epsilon}{\pi} \frac{\Gamma_{\ell, L} f_{L}(\epsilon)+\Gamma_{\ell, R} f_{R}(\epsilon)}{\Gamma_{\ell, L}+\Gamma_{\ell, R}} \operatorname{Im} G_{\ell, \ell}^{r}(\epsilon)
\end{gathered}
$$

The values of $N_{\ell, \sigma}$ and $c_{\ell}$ are restricted between 0 and 1 . The expression of two particle retarded Green function of Eq. (6) is

$$
G_{\ell, \ell}^{r}(\epsilon)=N_{\ell-\sigma} \sum_{m=1}^{3^{n-1}} \frac{p_{m}}{\epsilon-E_{\ell}-U_{\ell}-\Pi_{m}+i \Gamma_{\ell}} .
$$

\section{RESULTS AND DISCUSSION}

Although the thermal rectification effect of a single QD junction with two levels has been theoretically investigated in ref.[17], it is difficult to realize the physical condition of asymmetrical coupling between the QD and the electrodes, where the excited state states have symmetrical tunneling rates, but the ground state has an asymmetrical tunneling rate. Meanwhile, ref[17] did not take into account the crucial intralevel Coulomb interactions. To determine the direction-dependent heat current, we considered two sets of parameters (the cases of $T_{L}>T_{R}$ and $T_{R}>T_{L}$ ): (a) is $\mu_{L}=E_{F}+e \Delta V$, $\mu_{R}=E_{F}, T_{L}=T_{0}+\Delta T$, and $T_{R}=T_{0}$, and (b) is $\mu_{L}=E_{F}, \mu_{R}=E_{F}-e \Delta V, T_{L}=T_{0}$ and $T_{R}=$ $T_{0}+\Delta T$. The average temperature of two side electrodes is $\left(T_{L}+T_{R}\right) / 2=T_{0}+\Delta T / 2$, which is increasing steadily with $\Delta T$. Such a physical condition for average temperature is more readily realized than that of reference [17] 
from experimental point of view.[24] A functional thermal rectifier requires a good thermal conductance for the case of $T_{L}>T_{R}$, but a thermal insulator for the case of $T_{R}>T_{L}$. Based on Eqs. (2) and (3), a QD thermal rectifier requires not only highly asymmetrical coupling strengthes between the QDs and the electrodes, but also strong electron Coulomb interactions between the dots. To investigate the thermal rectification behavior, we numerically solved Eqs. (2) and (3) for three QD junctions for using various system parameters.

The energy level of dot $\ell$ is $E_{\ell}=E_{F}+\alpha_{\ell} \Delta E$, where $-1 \leq \alpha_{\ell} \leq 1$. The term $\Delta E=200 \Gamma$ represents the energy level fluctuation of QDs. For simplicity, the intradot Coulomb interactions $U_{\ell}=600 \Gamma$ are fixed, although the QD size fluctuation leads to the intradot Coulomb interaction variation. According to Eq. (3), the direction-dependent heat currents are attributed to $\Delta V$ and $N_{\ell, \sigma}=N_{\ell,-\sigma}=N_{\ell}$. The Seebeck effect for the open circuit $\left(J_{e}=0\right)$ determines the electrochemical potential $e \Delta V$. This electrochemical potential forms in response to the current generated by the temperature gradient. This electrochemical potential is the Seebeck voltage (Seebeck effect). The thermal power is $S=\Delta V / \Delta T$. Once $\Delta V$ is solved, we then use Eq. (3) to compute the heat current.

Figure. 2 shows the direction-dependent heat current $(\mathrm{Q})$ and rectification efficiency $\left(\eta_{Q}\right)$ as functions of $\Delta T$ for various values of $\Gamma_{A R}$, while keeping $\Gamma_{B(C), L}=$ $\Gamma_{B(C), R}=\Gamma$. We adopted the interdot Coulomb interactions $U_{\ell, j}=300 \Gamma$ and $k_{B} T_{0}=25 \Gamma$. The energy levels of $\operatorname{dots} \mathrm{A}, \mathrm{B}$, and $\mathrm{C}$ were chosen to be $E_{A}=E_{F}-\Delta E / 5$, $E_{B}=E_{F}+2 \Delta E / 5$ and $E_{C}=E_{F}+\Delta E / 5$. The heat currents have positive and negative signs corresponding for the cases of $T_{L}>T_{R}$ and $T_{R}>T_{L}$, respectively. The former is the heat current from the left electrode to the right electrode $Q_{L R}$, while the later is the heat current from the right electrode to the left electrode $Q_{R L}$. The rectification efficiency is $\eta_{Q}=\left(Q_{L R}(\Delta T)-\right.$ $\left.\left|Q_{R L}(\Delta T)\right|\right) / Q_{L R}(\Delta T)$. The rectification effects of heat currents in Fig. 2(a) are clearly distinguishable from Fig. 2 (b). When $\operatorname{dot} \mathrm{A}$ is fully blocked $\left(\Gamma_{A R}=0\right)$, the QD junction exhibits a much higher $\eta_{Q}$ and the heat currents decrease dramatically. This serious suppression implies that the heat currents through dots B and C are small because their resonant energy levels are far away from the Fermi energy level due to a large $U_{A C}$ and $U_{A B}$. The results in Fig. 2 indicate that precisely manipulating the coupling strengthes between the dots and the electrodes is crucial to the optimization of thermal rectifiers.

Figure. 2 illustrates the homogenous interdot Coulomb interactions. However it is difficult to achieve homogenous interdot Coulomb interactions in the multiple QD layout. The interdot Coulomb interaction fluctuation mainly arises from the size variation and the inhomogenous position distribution. Therefore, we examined the variation effect of $U_{A C}$ on heat currents. Figure. 3 shows the heat current and rectification efficiency as functions of $\Delta T$ for various values of $U_{A C}$ at $\Gamma_{A R}=0$ and $\Gamma_{A L}=2 \Gamma$. The other parameters are the same as those in
Fig. 2. In this case, large interdot Coulomb interactions suppress the heat currents. This behavior is similar to the Coulomb blockade effect on the charge current. ${ }^{22,23}$ Nevertheless, a strong $U_{A C}$ leads to higher rectification efficiency. Therefore, a higher efficient thermal rectification effect appears in the high density QD system. In the such a case of strong intradot and interdot Coulomb interactions, the charge current exhibits clear plateaus with respect to applied voltage. However, the structure of heat currents in Fig. 3 does not exhibit these plateaus with respect to temperature bias. This is because temperature bias broadens the electron distribution. [22]

In Figures (2) and (3) we illustrate the case of $E_{C}<$ $E_{B}$. Figure. 4 shows that $E_{C}$ influences the heat current and $\eta_{Q}$. The other parameters are the same as those in Fig. 3. When $E_{C}$ changes from $E_{C}=$ $E_{F}+\Delta E / 5$ to $E_{C}=E_{F}+4 \Delta E / 5$, the heat current for $E_{C}=E_{F}+2 \Delta E / 5$ is less than that for $E_{C}=$ $E_{F}+3 \Delta E / 5$. This indicates that the separation between the energy levels of QDs and the Fermi energy level is not a unique factor to influence the heat current magnitude. When $E_{C}$ increases to $E_{C}=E_{F}+4 \Delta E / 5$ from $E_{C}=E_{F}+3 \Delta E / 5$, the heat currents and rectification efficiency change slightly. All of these complicated behaviors are the result of the very nonlinear relationship between the heat currents and electrochemical potentials, which are strongly influenced by the electron Coulomb interactions and the energy levels of each QD.

The following expression of heat current, $Q=Q_{B}+$ $Q_{C}$, further clarifies the results of Fig. 4:

$$
\begin{aligned}
Q_{B} /\left(\gamma_{B} \pi\right) & =\left(1-N_{B}\right) *\left(( 1 - 2 N _ { A } ) ( E _ { B } - E _ { F } ) f _ { L R } \left(E_{B}(4)\right.\right. \\
& +2 N_{A}\left(E_{B}+U_{A B}-E_{F}\right) * f_{L R}\left(E_{B}+U_{A B}\right) \\
Q_{C} /\left(\gamma_{C} \pi\right) & =\left(1-N_{C}\right) *\left(\left(1-2 N_{A}\right)\left(E_{C}-E_{F}\right) f_{L R}\left(E_{C}\right)\right. \\
& +2 N_{A}\left(E_{C}+U_{A C}-E_{F}\right) * f_{L R}\left(E_{C}+U_{A C}\right)(5)
\end{aligned}
$$

Equations. (7) and (8) are the results of setting $c_{\ell}$ to zero due to very large intradot Coulomb interactions. Further, a delta function replaces the Lorentzian function of resonant channels for $\Gamma \ll k_{B} T_{0}$ in Eq. (3). In addition, Eqs. (7) and (8) only consider the interdot Coulomb interactions $U_{A B}$ and $U_{A C}$ since there is one particle occupation in dot A. As for $N_{A}, N_{B}, N_{C}$ and $e \Delta V$ in Eqs. (7) and (8), we use full numerical solution (Eqs (2),(5) and (6)) to solve them. Figure. 5 shows the heat current as functions of $\Delta T$ for $E_{C}=E_{F}+3 \Delta E / 5$. The other parameters are the same as those in Fig.4. The lot lines given in Eqs. (7) and (8) match the full solutions very well. $Q_{L R}$ and $Q_{R L}$ are the nonlinear functions of $\Delta T$. Figure. $5(\mathrm{~b})$ shows the $Q_{B}$ and $Q_{C}$ calculated by results of Eqs. (7) and (8) for $Q_{L R}$ and $Q_{R L}$. Based on Fig. $5(\mathrm{~b}), Q_{C}$ is the primary determinant of the heat currents of Fig. 5(a). The factors of $N_{A}$ and $f_{L R}$ influence the behavior of $Q_{L R}$ with respect to $\Delta T$. Only the factor of $f_{L R}$ influences the behavior of $Q_{R L}$. This is because of $\Delta T$-independent $N_{A}=0.45$ in the case of $Q_{R L}$. So far, we have analyzed the heat current and $\eta_{Q}$. From experimental point of view, it is easy to measure 
the electrochemical potential yielded by the temperature bias although it is not straightforwardly related to the heat current in this system.

Figure. 6 shows the electrochemical potential of different values of $k_{B} T_{0}$. The other parameters are the same as those in Fig. 5. The dot and dashed lines represent the linear functions of $\Delta T$, whereas the solid lines are nonlinear with respect to $\Delta T$. Although the asymmetrical electrochemical potentials (Fig. (6a) and (6b)) reveal some information about thermal rectification, it is difficult to determine the rectification efficiency from the measurement of electrochemical potentials. This is true for both linear and nonlinear responses due to the very nonlinear relation between $\mathrm{Q}$ and $\Delta V$. Therefore, it is difficult to judge the thermal rectification effect from the experimental results done in ref[16]. The large electrochemical potential created by the temperature bias $(\Delta T)$ is useful for generating electrical powers. The results of Fig. 6 also imply that the charge current rectification with respect to temperature appears in the closed circuit $\left(J_{e} \neq 0\right)$. An electrochemical potential is an essential quantity for charge carriers to deliver heat current, it is different from the phonon carriers in a phonon junction system. ${ }^{12-15}$ To further illustrate the strong nonlinear relationship between electrochemical potential and heat current, we show the heat current and rectification efficiency in Fig. 7, where the curves exhibit one to one correspondence with those in Fig. 6. We note that $\eta_{Q}$ is enhanced when temperature $k_{B} T_{0}$ decreases. A comparison of Fig. 6 and Fig. 7 demonstrates the strong nonlinear relationship between $\mathrm{Q}$ and $\Delta V$.

Finally, figure 8 calculates the thermal power as a function of $k_{B} T_{0}$ for different values of $\Delta T$. The other parameters are the same as those in Fig. 5. As expected, the temperature bias has a significant influence on ther- mal powers at low $k_{B} T_{0}$, but not at high temperatures. Note that the thermal power values increase with increasing temperature bias. Thermal power plays a significant role in determining the figure of merit.[10] Some thermal devices such as solid state coolers are in the nonlinear response regime. ${ }^{2}$ Therefore, the previous studies of linear response regime underestimate the thermal power values when $\Delta T / T_{0} \geq 1 .[10,25]$

\section{CONCLUSIONS}

This theoretical study reports the thermal rectification effects (TRE) of multiple QDs embedded into an amorphous insulator (phonon glass) with low heat conductivity. Results show that the asymmetrical tunneling rates and strong interdot Coulomb interactions significantly influence the TRE. Recent research indicates that it is possible to precisely manipulate the size and position of multiple germanium QDs in $\mathrm{SiO}_{2}$ amorphous insulator.[26] This experiment indicates that it is possible to realize the system shown in Fig. 1 to examine the thermal rectification at high temperatures. To avoid the phonon heat current, which can seriously suppress the heat rectification effect arising from electrons, scantunneling- microscopes (STMs) can replace one of the electrodes since the vacuum layer between the STM and amorphous insulator can blockade the phonon heat current.[27]

\section{Acknowledgments}

This work was supported by the National Science Council of the Republic of China under Contract Nos. NSC 97-2112-M-008-017-MY2 and NSC 98-2112-M-001022-MY3.
[1]A. J. Minnich, M. S. Dresselhaus, Z. F. Ren and G. Chen, Energy Environ Sci, 2, (2009) 466.

[2]G. Mahan, B. Sales and J. Sharp, Physics Today, 50, (1997) 42.

[3]R. Venkatasubramanian, E. Siivola,T. Colpitts,B. O’Quinn, Nature 413, (2001) 597.

[4]A. I. Boukai, Y. Bunimovich, J. Tahir-Kheli, J. K. Yu, W. A. Goddard III and J. R. Heath, Nature, 451, (2008) 168.

[5]T. C. Harman, P. J. Taylor, M. P. Walsh, B. E. LaForge, Science 297, (2002) 2229.

[6]K. F. Hsu,S. Loo,F. Guo,W. Chen,J. S. Dyck,C. Uher, T. Hogan, E. K. Polychroniadis,M. G. Kanatzidis, Science 303, (2004) 818.

[7]A. Majumdar, Science 303, (2004) 777.

[8]G. Chen, M. S. Dresselhaus, G. Dresselhaus, J. P. Fleurial and T. Caillat, International Materials Reviews, 48, (2003) 45.

[9]Y. M. Lin and M. S. Dresselhaus, Phys. Rev. B 68, (2003) 075304 .

[10]D. M. T. Kuo, Jpn. J. Appl. Phys. 48 (2009) 125005.
[11]C. Starr, J. Appl. Phys. 7, (1936) 15.

[12]M. Terraneo, M. Peyrard, G. Casati, Phys. Rev. Lett. 88, (2002) 094302.

[13]Baowen Li, L. Wang and G. Casati, Phys. Rev. Lett. 93, (2004) 184301.

[14]B. Hu, L. Yang and Y. Zhang, Phys. Rev. Lett. 97, (2006) 124302.

[15]G. Casati, C. Mejia-Monasterio and T. Prosen, Phys. Rev. Lett. 98, (2007) 104302.

[16]R. Scheibner, M. Konig, D. Reuter, A. D. Wieck, C. Gould, H. Buhmann and L. W. Molenkamp, New. J. Phys. 10, (2008) 083016.

[17]X. O. Chen, B. Dong and X. L. Lei, Chin. Phys. Lett. 25. (2008) 3032 .

[18]D. M. T. Kuo and Y. C. Chang, Phys. Rev. Lett. 99, (2007) 086803.

[19]H. Haug and A. P. Jauho, Quantum Kinetics in Transport and Optics of Semiconductors (Springer, Heidelberg, 1996).

[20]M. Krawiec and K. I. Wysokinski, Phys. Rev. B 75, (2007) 155330. 
[21]B. Dong and X. L. Lei, J. Phys. Condens. Matter 14 (2002) 11747.

[22]D. M. T. Kuo, Jpn. J. Appl. Phys. 47, (2008) 8291.

[23]Y. C. Chang and D. M. T. Kuo, Phys. Rev. B 77, (2008) 245412.

[24]D. Segal, Phys. Rev. B 73, (2006) 205415.

[25]P. Murphy, S. Mukerjee and J. Moore, Phys. Rev. B 78, (2008) 161406.

[26]K. H. Chen, C. Y. Chien and P. W. Li, Nanotechnology 21, (2010) 055302.

[27]M. Krawiec and M. Jalochowski, Physica Status Solidi B. 244, (2007) 2464.

\section{Figure Captions}

Fig. 1. Schematic representation of multiple semiconductor quantum dots embedded into an amorphous insulator with low heat conductivity sandwiched between metallic electrodes.

Fig. 2. Heat current and rectification efficiency as functions of $\Delta T$ for the tunneling rate variation of dot A. The heat currents are in the units of $Q_{0}=\Gamma^{2} /(2 h)$. Such units are used through out this article.
Fig. 3. Heat current and rectification efficiency as functions of $\Delta T$ for different interdot Coulomb interactions of $U_{A C}$ at $\Gamma_{A R}=0$ and $\Gamma_{A L}=2 \Gamma$. The other parameters are the same as those in Fig. 2.

Fig. 4. Heat current and rectification efficiency as functions of $\Delta T$ for different energy levels of $E_{C}$ and $U_{A C}=300 \Gamma$. The other parameters are the same as those in Fig. 3.

Fig. 5. Heat current as functions of $\Delta T$ for $E_{C}=$ $E_{F}+3 \Delta E / 5$. The Other parameters are the same as those in Fig. 4.

Fig. 6. Electrochemical potential as functions of $\Delta T$ for different values of $k_{B} T_{0}$. The other parameters are the same as those in Fig. 5.

Fig. 7. Heat current and rectification efficiency as functions of $\Delta T$ for different values of $k_{B} T_{0}$. The curves are one to one correspondence to those of Fig. 6

Fig. 8. Thermal power as functions of $k_{B} T_{0}$ for the different values of $\Delta T$. The other parameters are the same as those in Fig. 5. 


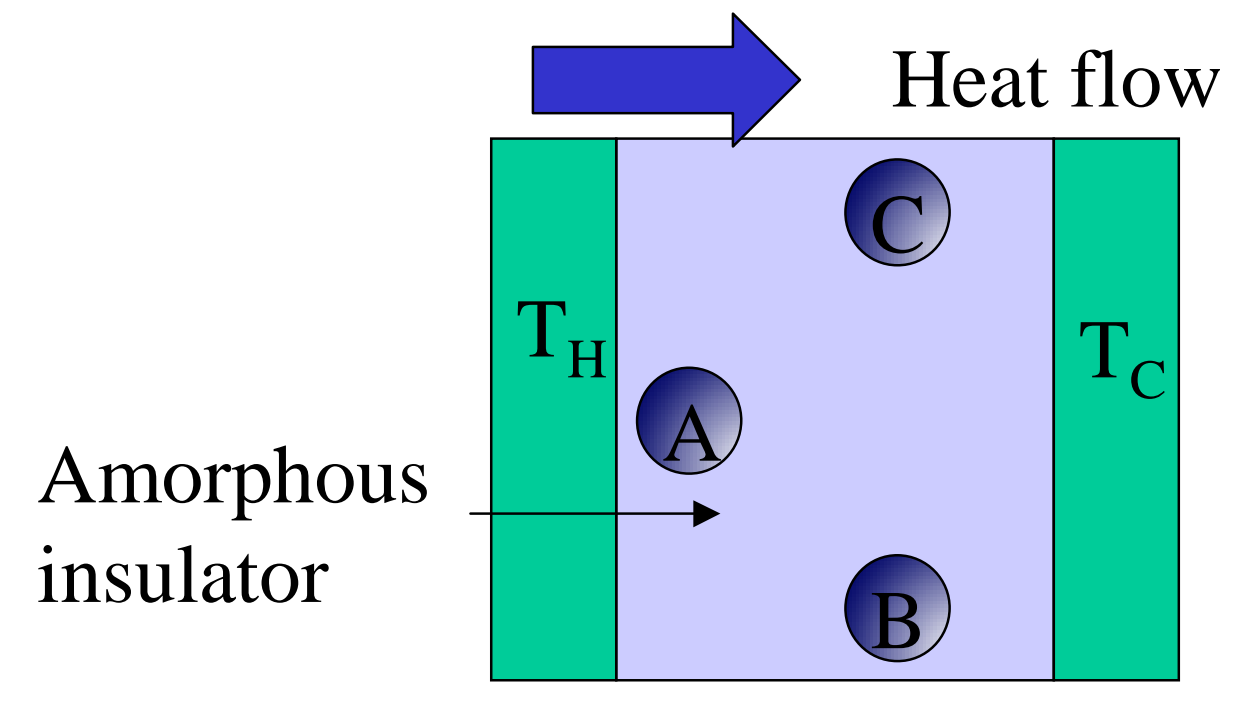

(a)

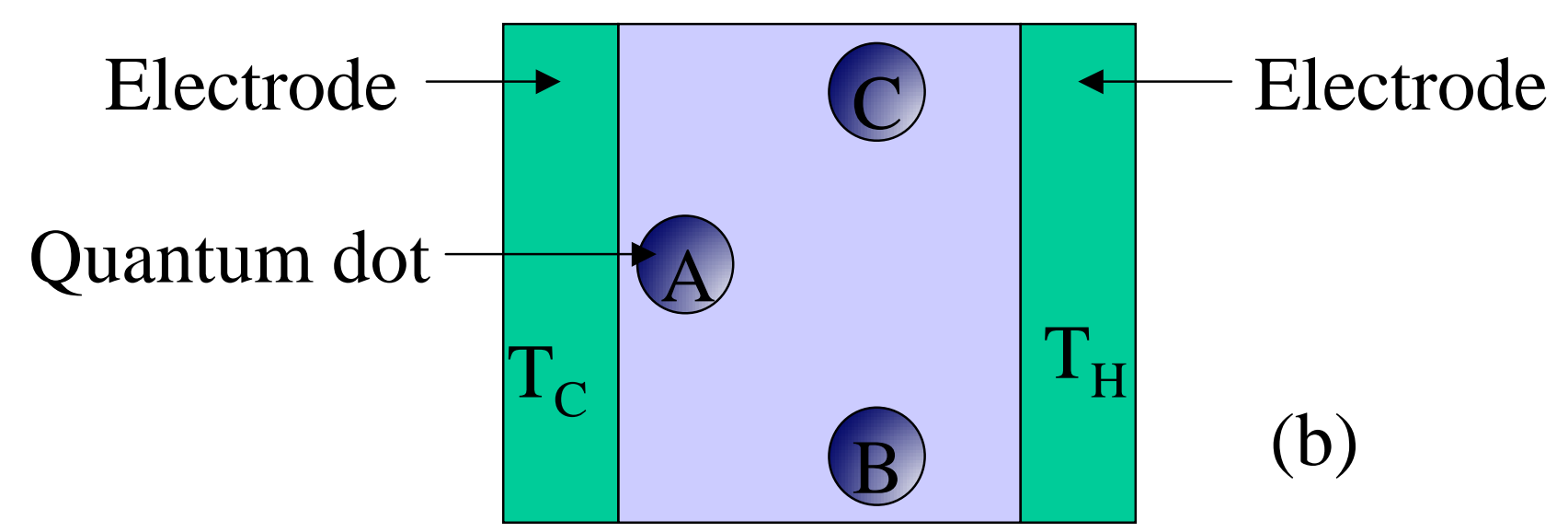

Heat flow

Fig1 


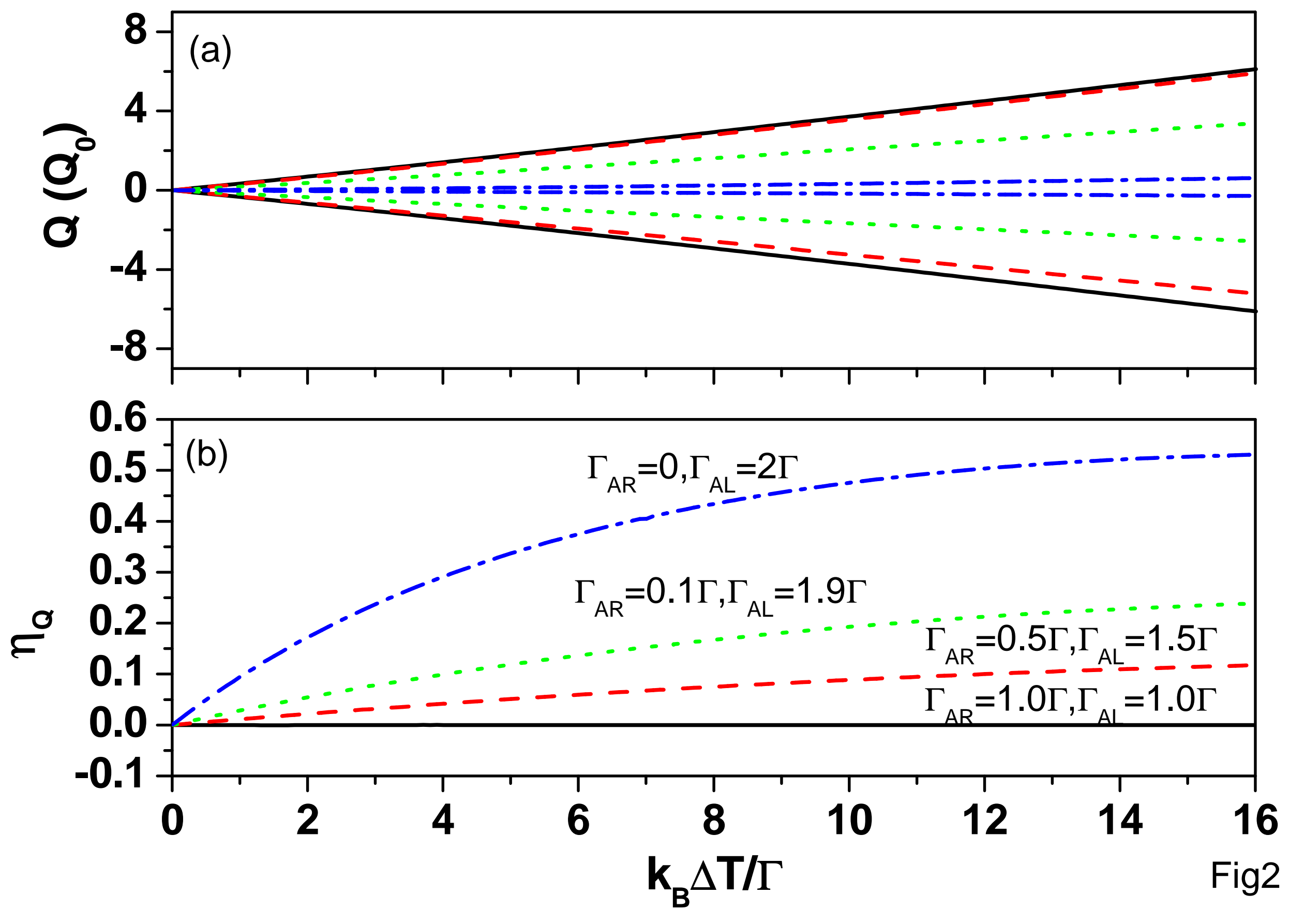



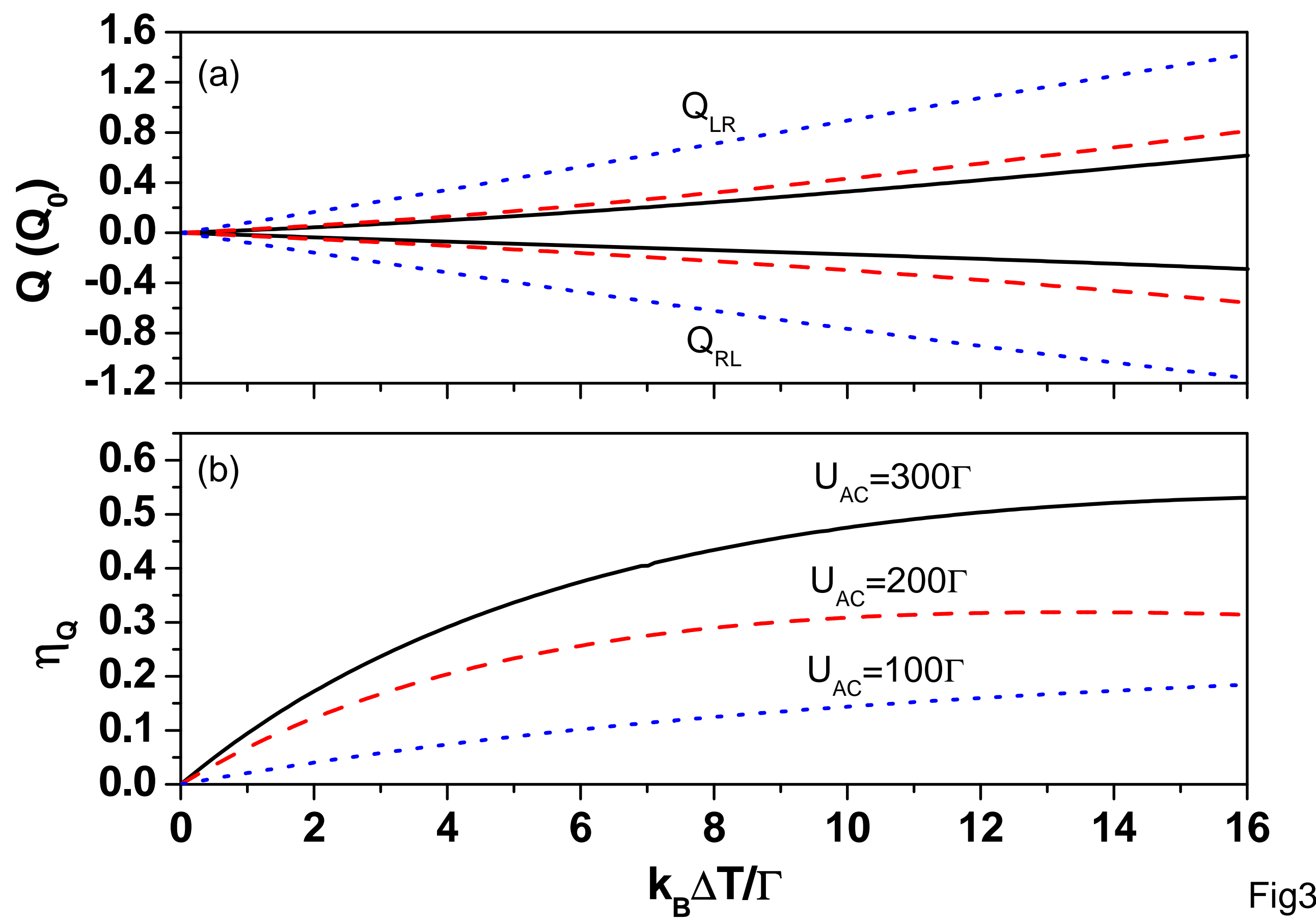

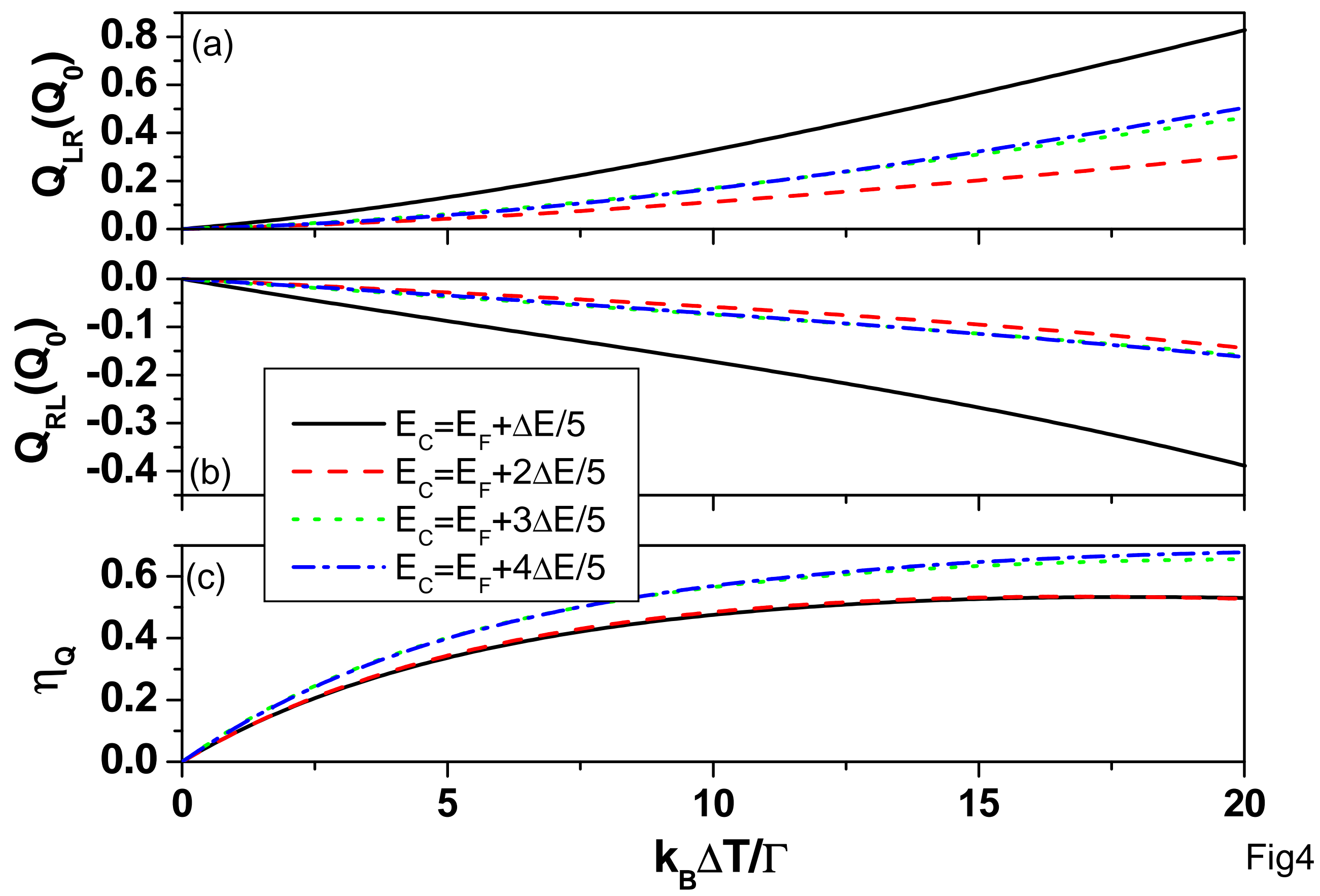

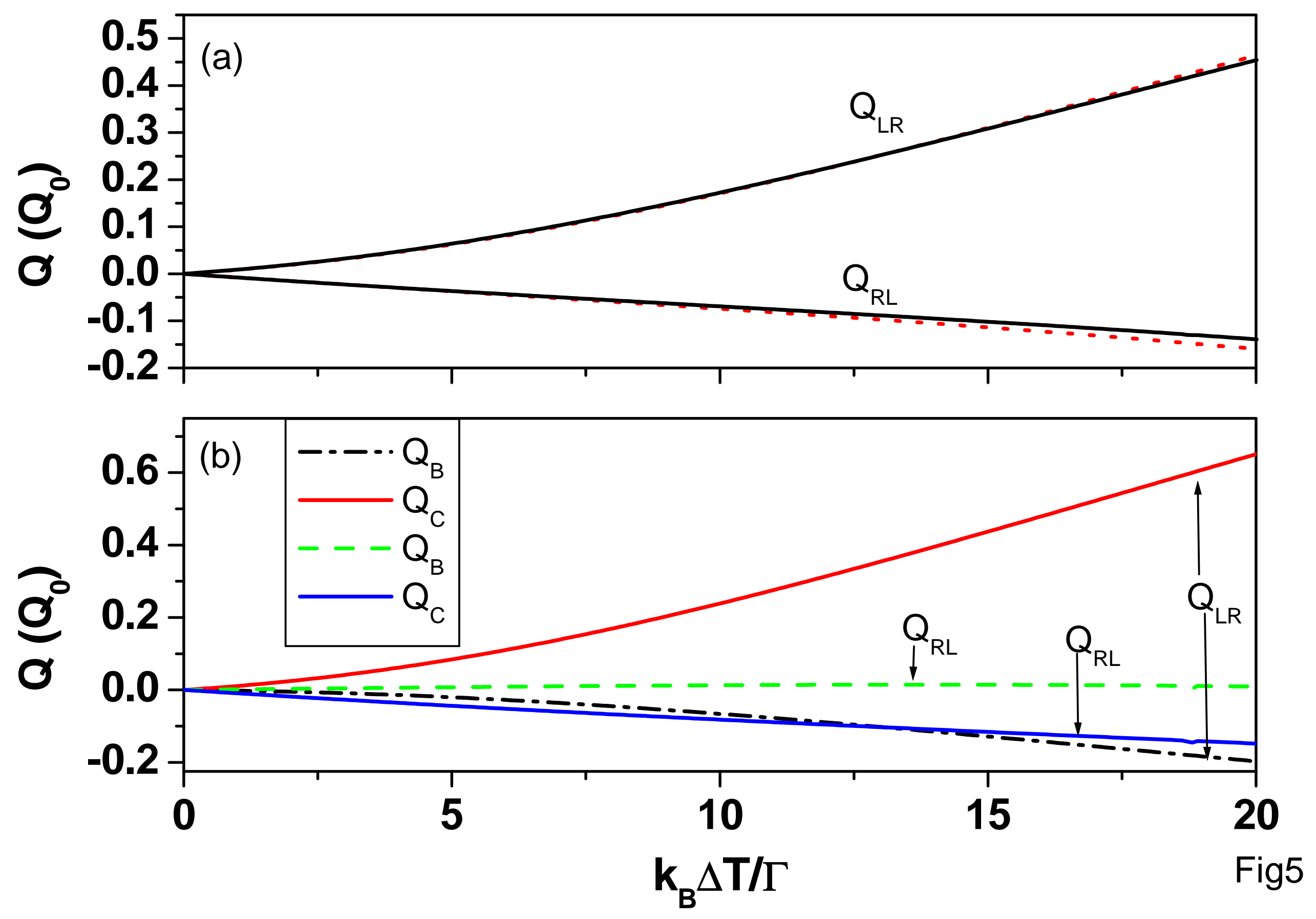


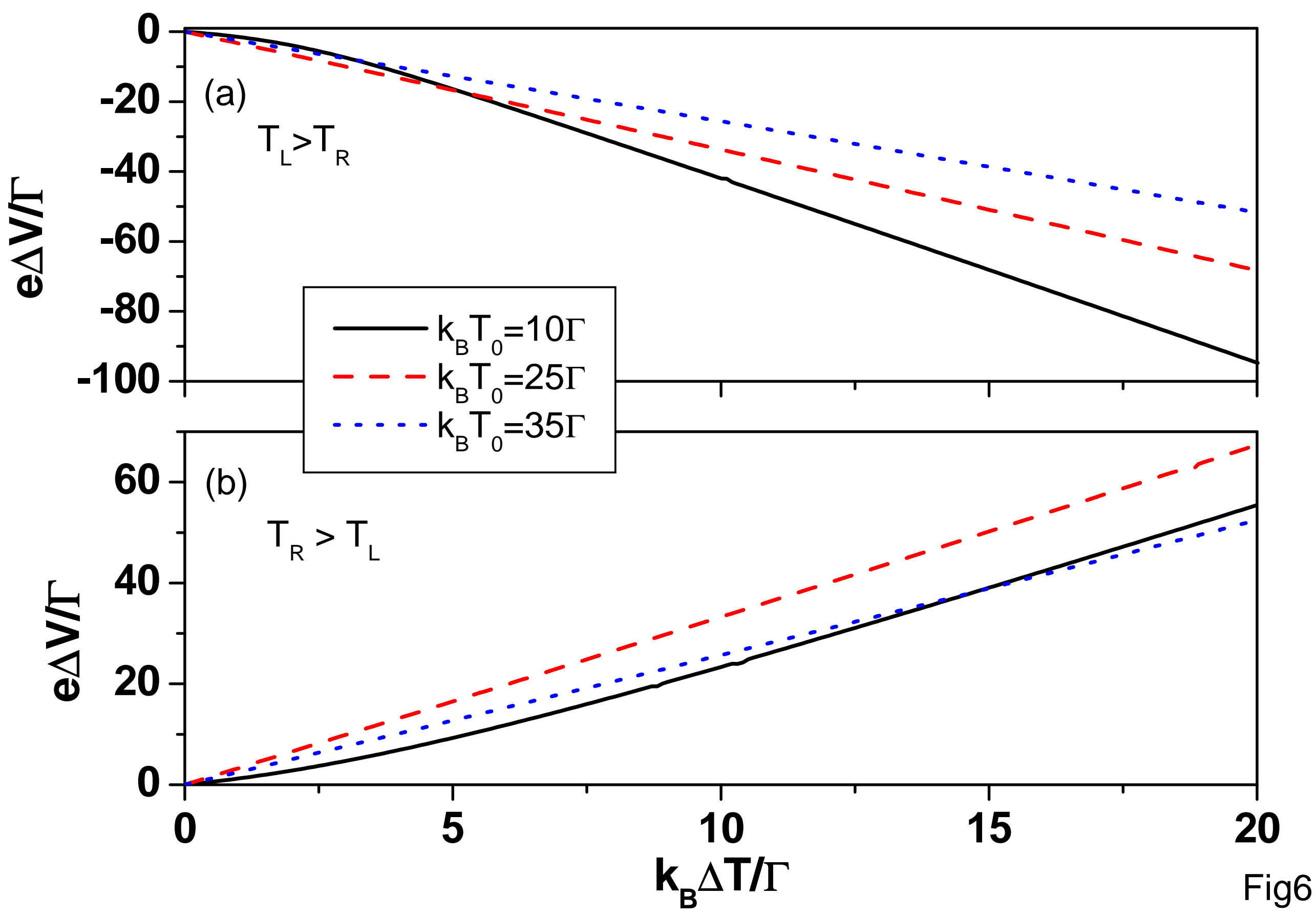




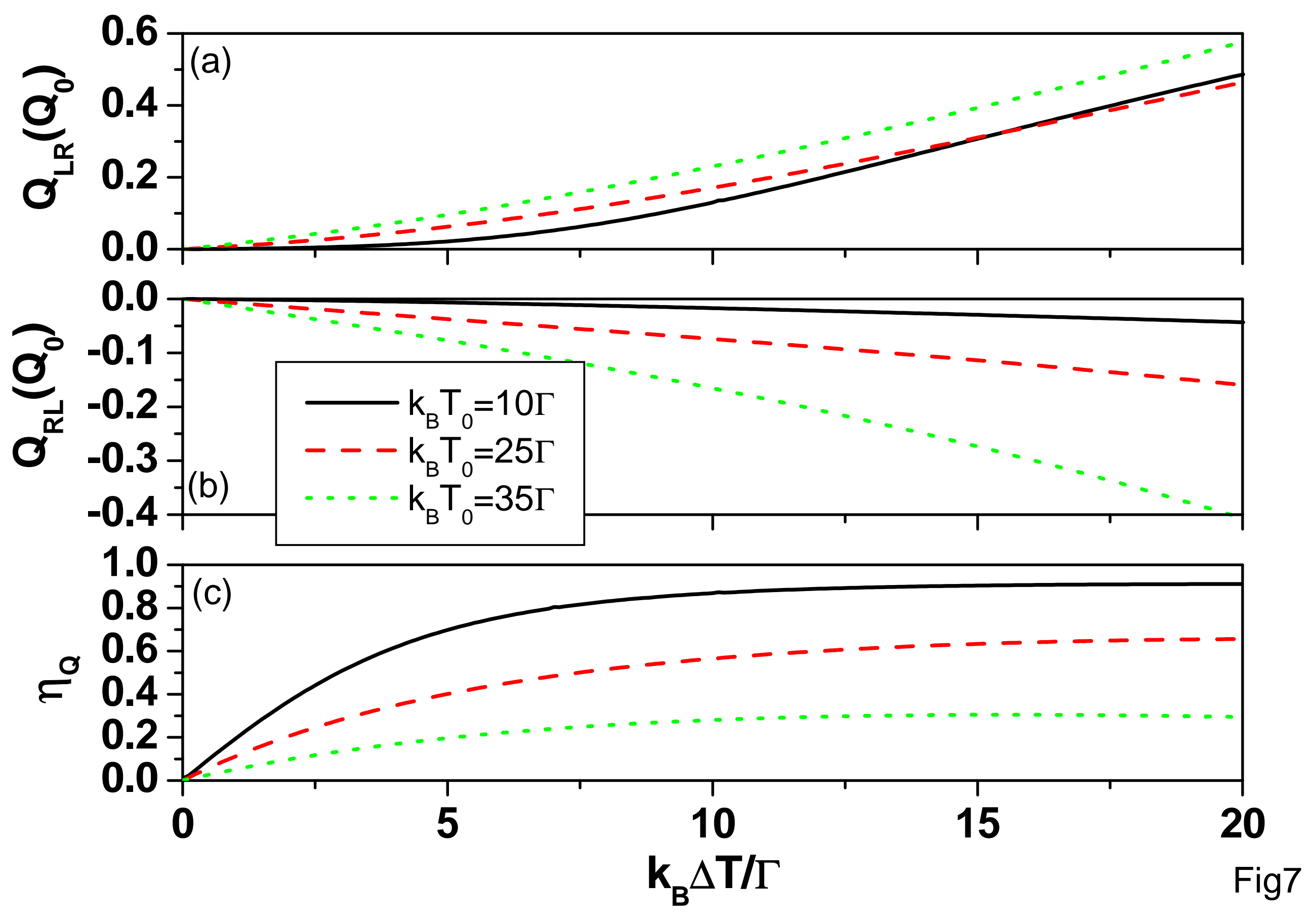



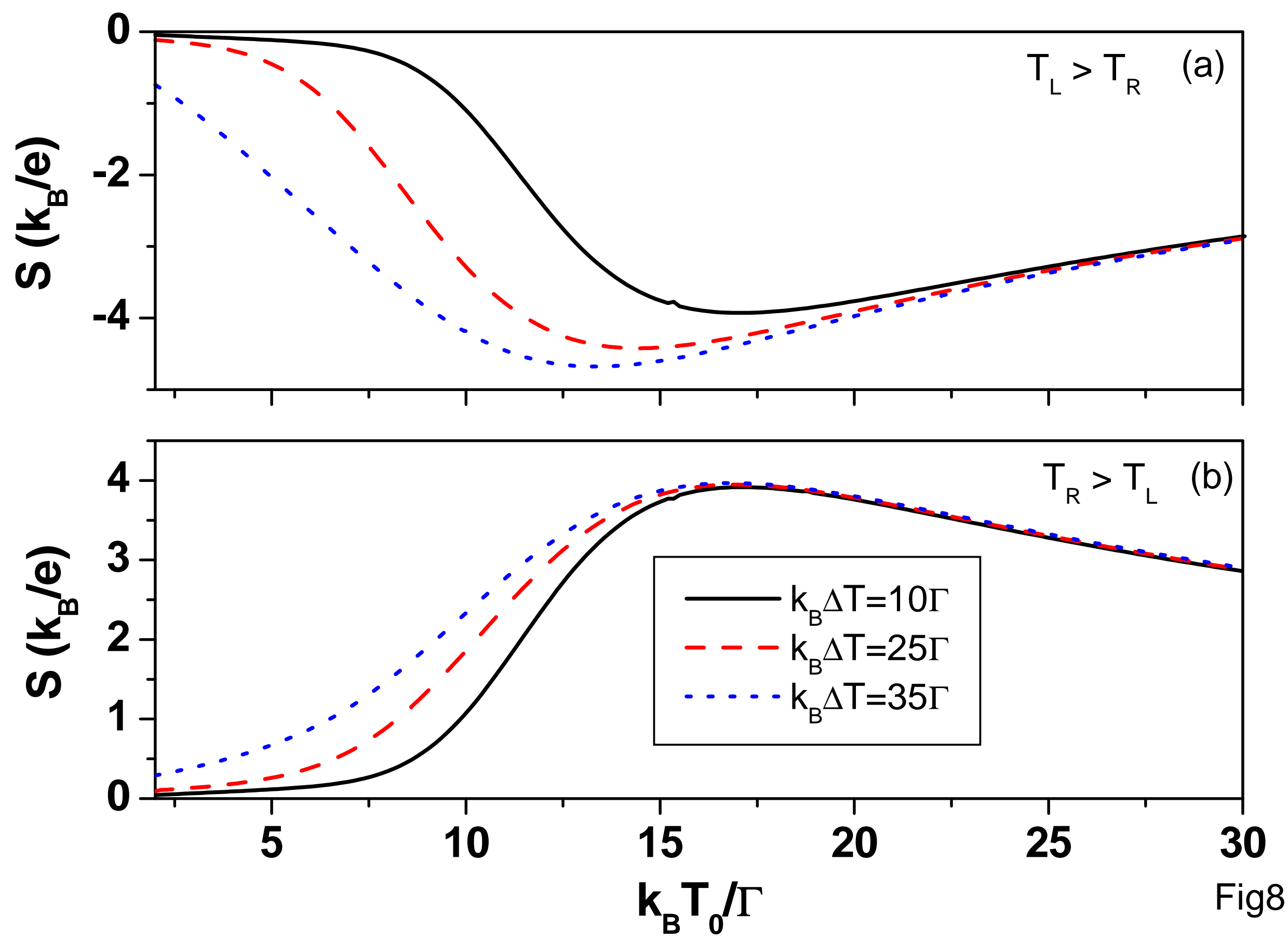